\title{
On solutions of integral equations with analytic kernels and rotations
}

\author{
by Nguyen Van Mau and Nguyen Minh Tuan (Hanoi)
}

\begin{abstract}
We deal with a class of integral equations on the unit circle in the complex plane with a regular part and with rotations of the form

$$
x(t)+a(t)(T x)(t)=b(t),
$$

where $T=M_{n_{1}, k_{1}} \ldots M_{n_{m}, k_{m}}$ and $M_{n_{j}, k_{j}}$ are of the form (3) below. We prove that under some assumptions on analytic continuation of the given functions, $(*)$ is a singular integral equation for $m$ odd and is a Fredholm equation for $m$ even. Further, we prove that $T$ is an algebraic operator with characteristic polynomial $P_{T}(t)=t^{3}-t$. By means of the Riemann boundary value problems, we give an algebraic method to obtain all solutions of equation $(*)$ in closed form.
\end{abstract}

1. Algebraic characterizations of integral operators with rotations. Let $\Gamma=\{t:|t|=1\}, D^{+}=\{t:|t|<1\}$ and let $X=H^{\mu}(\Gamma)(0<$ $\mu<1)$. Consider the following operators in $X$ :

$$
\begin{aligned}
(S x)(t) & =\frac{1}{\pi i} \int_{\Gamma} \frac{1}{s-t} x(s) d s \\
\left(S_{n, k} x\right)(t) & =\frac{1}{\pi i} \int_{\Gamma} \frac{s^{n-1-k} t^{k}}{s^{n}-t^{n}} x(s) d s \\
\left(M_{n, k} x\right)(t) & =\frac{1}{\pi i} \int_{\Gamma} \frac{s^{n-1-k} t^{k} \mathcal{M}_{n}(s, t)}{s^{n}-t^{n}} x(s) d s
\end{aligned}
$$

where $n, k \in \mathbb{N}, n>1,0 \leq k \leq n-1$ and $\mathcal{M}_{n}(s, t)$ satisfies the Hölder condition with respect to $(s, t) \in \Gamma \times \Gamma$. Let $\varepsilon_{n, 1}=e^{2 \pi i / n}, \varepsilon_{n, j}=\varepsilon_{n, 1}^{j}, j=$ $1, \ldots, n$. Consider the rotation operators $\left(W_{n} x\right)(t)=x\left(\varepsilon_{n, 1} t\right)$. For $j \in$

1991 Mathematics Subject Classification: 47G05, 45G05, 45E05.

Key words and phrases: integral operators, singular integral equations, algebraic operators, Riemann boundary value problems. 
$\{1, \ldots, n\}$, write

$$
P=\frac{1}{2}(I+S), \quad Q=\frac{1}{2}(I-S), \quad P_{n, j}=\sum_{k=1}^{n} \varepsilon_{n, j}^{n-1-k} W_{n}^{k+1} .
$$

It is easy to check that $X=X^{+} \oplus X^{-}$and $X=\bigoplus_{j=1}^{n} X_{n, j}$, where $X^{+}=$ $P X, X^{-}=Q X$ and $X_{n, j}=P_{n, j} X$ for $j \in\{1, \ldots, n\}$.

Lemma 1. Let $P, Q$ and $P_{n, j}$ be defined by (4). Then

$$
P_{n, j} X^{+} \subset X^{+}, \quad P_{n, j} X^{-} \subset X^{-}
$$

and

$$
P X_{n, j} \subset X_{n, j}, \quad Q X_{n, j} \subset X_{n, j} \quad(j \in\{1, \ldots, n\}) .
$$

Proof. This follows immediately from the equalities

$$
S W_{n}=W_{n} S, \quad S_{n, k} S=S S_{n, k}, \quad S_{n, k} W_{n}=W_{n} S_{n, k},
$$

for all $k \in\{0, \ldots, n-1\}$.

Lemma 2 ([2]-[3]). $S_{n, k}=S P_{n, k}=P_{n, k} S$ for all $k \in\{0, \ldots, n-1\}$, where we set $P_{n, 0}=P_{n, n}$.

Lemma 3. Suppose that $\mathcal{M}_{n}(s, t)$ is invariant with respect to the rotation operator $W_{n}$, i.e. $\mathcal{M}_{n}\left(\varepsilon_{n, 1} s, t\right)=\mathcal{M}_{n}\left(s, \varepsilon_{n, 1} t\right)=\mathcal{M}_{n}(s, t)$. Suppose, moreover, that $\mathcal{M}_{n}(t, t)=1$ for all $t \in \Gamma$. Then

(5) $\quad M_{n, k}=S_{n, k}+N_{n} P_{n, k}, \quad N_{n} P_{n, k}=P_{n, k} N_{n} \quad$ for all $k \in\{1, \ldots, n\}$,

where

$$
\left(N_{n} x\right)(t)=\frac{1}{\pi i} \int_{\Gamma} \mathcal{N}_{n}(s, t) x(s) d s, \quad \mathcal{N}_{n}(s, t)=\frac{\mathcal{M}_{n}(s, t)-1}{s-t} .
$$

Proof. The assumptions on $\mathcal{M}_{n}(s, t)$ imply the equalities

$$
\begin{aligned}
\left(M_{n, k} x\right)(t) & =\left(S_{n, k} x\right)(t)+\frac{1}{\pi i} \int_{\Gamma} \frac{s^{n-1-k} t^{k}}{s^{n}-t^{n}}\left(\mathcal{M}_{n}(s, t)-1\right) x(s) d s \\
& =\left(S_{n, k} x\right)(t)+\sum_{j=1}^{n} \varepsilon_{k}^{n-1-j}\left(\frac{1}{\pi i} \int_{\Gamma} \frac{\mathcal{M}_{n}(s, t)-1}{s-\varepsilon_{j} t} x(s) d s\right) \\
& =\left(S_{n, k} x\right)(t)+\frac{1}{\pi i} \int_{\Gamma} \mathcal{N}_{n}(s, t)\left(P_{n, k} x\right)(s) d s,
\end{aligned}
$$

which gives the first equality of the formula (5). The second equality of (5) immediately follows from the assumption that $\mathcal{M}_{n}(s, t)$ is invariant with respect to $W_{n}$.

TheOREM 1. Let $n>1$ and $m \in\{0, \ldots, n-1\}$. Suppose that $\mathcal{M}_{n}(s, t)$ satisfy all assumptions of Lemma 3 and admit an analytic continuation in 
both variables into $D^{+}$. Write

$$
M=\sum_{k=0}^{m} M_{n, k}
$$

where $M_{n, k}$ is of the form (3). Then $M$ is an algebraic operator and $M^{3}=M$.

Proof. From the assumptions on $\mathcal{M}_{n}(s, t)$, the function $\mathcal{N}_{n}(s, t)=$ $\left(\mathcal{M}_{n}(s, t)-1\right)(s-t)^{-1}$ is continuous in $(s, t) \in \Gamma \times \Gamma$ and admits an analytic continuation in both variables into $D^{+}$. Hence, the Cauchy integral theorem gives

$$
N_{n}^{2}=0, \quad S N_{n}=N_{n}, \quad N_{n} S=-N_{n} .
$$

On the other hand, since $S^{2}=I, S_{n, k}^{2}=P_{n, k}$ and $P_{n, k} P_{n, j}=\delta_{k j} P_{n, j}$ we find

and

$$
M_{n, k}^{2}=P_{n, k}, \quad M_{n, k}^{3}=M_{n, k}, \quad M_{n, k} M_{n, j}=\delta_{k j} M_{n, j}
$$

$$
M^{3}=\sum_{k=0}^{m} M_{n, k}^{3}=\sum_{k=0}^{m} M_{n, k}=M,
$$

which gives the proof.

TheOREM 2. Let $m \in \mathbb{N}^{+}$. Write

$$
T=M_{n_{1}, k_{1}} M_{n_{2}, k_{2}} \ldots M_{n_{m}, k_{m}},
$$

where $M_{n_{j}, k_{j}}(j \in\{1, \ldots, m\})$ are of the form (3). Suppose that $\mathcal{M}_{n_{j}}(t, t)$ $=1$, and $\mathcal{M}_{n_{j}}(s, t)(j \in\{1, \ldots, m\})$ admit an analytic continuation in both variables into $D^{+}$and are invariant with respect to $W_{N}$, where $N=$ $\prod_{j=1}^{m} n_{j}$. Then $T$ is an algebraic operator and $T^{3}=T$.

Proof. Since every $\mathcal{M}_{n_{j}, k_{j}}(s, t)$ is invariant with respect to $W_{N}$, by Lemma 3, we conclude that

$$
M_{n_{\nu}, k_{\nu}} M_{n_{\mu}, k_{\mu}}=M_{n_{\mu}, k_{\mu}} M_{n_{\nu}, k_{\nu}} \quad \text { for all } \mu, \nu \in\{1, \ldots, m\} .
$$

Hence, the proof immediately follows from Theorem 1.

Remark 1. If $k, j \in\{0, \ldots, n\}$ and $k \neq j$ then $M_{n, k} M_{n, j}=0$ for $P_{n, k} P_{n, j}=0$. Hence, if there are $i, j \in\{1, \ldots, m\}$ and $i \neq j$ such that $n_{i}=n_{j}$ and $k_{i} \neq k_{j}$, then $T=0$. Therefore, in the sequel, we only deal with the cases $n_{i} \neq n_{j}$ for $i \neq j$.

Lemma 4. Let $l \in \mathbb{N}^{+}$. Suppose that $\mathcal{M}_{n_{j}}(s, t)(j \in\{1, \ldots, m\})$ satisfy all assumptions of Theorem 2. Then

$$
\prod_{k=1}^{m}\left(S+N_{n_{k}}\right)=I+\sum_{k=1}^{2 l}(-1)^{k} N_{n_{k}} \quad \text { for } m=2 l
$$


and

$$
\prod_{k=1}^{m}\left(S+N_{n_{k}}\right)=S+\sum_{k=1}^{2 l-1}(-1)^{k+1} N_{n_{k}} \quad \text { for } m=2 l-1 .
$$

Proof. Since $S N_{n_{2}}=N_{n_{2}}, N_{n_{1}} S=-N_{n_{1}}$ and $N_{n_{1}} N_{n_{2}}=0$ (see the proof of Theorem 2), for $l=1$ we have

$$
\left(S+N_{n_{1}}\right)\left(S+N_{n_{2}}\right)=I+S N_{n_{2}}+N_{n_{1}} S+N_{n_{1}} N_{n_{2}}=I+N_{n_{2}}-N_{n_{1}} .
$$

Hence, it is easy to check the formula (8) by induction on $l$.

Similarly, (9) is trivial for the case $l=1$. Suppose that (9) is true for $l=s$. Then for $l=s+1$, we find

$$
\begin{aligned}
\prod_{k=1}^{2 s+1}\left(S+N_{n_{k}}\right) & =\left(S+\sum_{k=1}^{2 s-1}(-1)^{k+1} N_{n_{k}}\right)\left(S+N_{n_{2 s}}\right)\left(S+N_{n_{2 s+1}}\right) \\
& =\left(S+\sum_{k=1}^{2 s-1}(-1)^{k+1} N_{n_{k}}\right)\left(I+N_{n_{2 s+1}}-N_{n_{2 s}}\right) \\
& =S+\sum_{k=1}^{2 s-1}(-1)^{k+1} N_{n_{k}}-S N_{n_{2 s}}+S N_{n_{2 s+1}} \\
& =S+\sum_{k=1}^{2 s+1}(-1)^{k+1} N_{n_{k}},
\end{aligned}
$$

which proves the formula (9).

2. Integral equations with rotations. In the sequel, for every fixed $a \in X$, we write $\left(K_{a} x\right)(t)=a(t) x(t)$.

Lemma 5. Let $k, j \in\{1, \ldots, n\}$ be fixed. Then for every $a \in X$ there exists an element $b \in X$ such that $K_{b} X_{j} \subset X_{k}$ and

$$
P_{n, k} K_{a} P_{n, j}=K_{b} P_{n, j} \quad \text { on } X .
$$

(Such a function $b(t)$ will be denoted by $a_{n k j}(t)$.)

Proof. Note that $P_{n, k}$ defined by (4) is a polynomial in $W_{n}$ with constant coefficients. On the other hand, we also have

$$
P_{n, j} P_{n, k}=\delta_{j k} P_{n, k}, \quad W_{n}^{l}=\sum_{k=1}^{n} \varepsilon_{n, k}^{l} P_{n, k} \quad \text { for all } l \in \mathbb{N} .
$$

Hence

$$
P_{n, k} K_{a} P_{n, j}=\sum_{l=1}^{n} \varepsilon_{n, k}^{n-1-l} W_{n}^{l+1} K_{a} P_{n, j}=\sum_{l=1}^{n} \varepsilon_{n, k}^{n-1-l} a\left(\varepsilon_{n, l+1} t\right) W^{l+1} P_{n, j}
$$




$$
\begin{aligned}
& =\sum_{l=1}^{n} \varepsilon_{n, k}^{n-1-l} a\left(\varepsilon_{n, l+1} t\right) \sum_{\nu=1}^{n} \varepsilon_{n, \nu}^{l+1} P_{n, \nu} P_{n, j} \\
& =\sum_{l=1}^{n} \varepsilon_{n, k}^{n-1-l} a\left(\varepsilon_{n, l+1} t\right) \varepsilon_{n, j}^{l+1} P_{n, j}=a_{n k j}(t) P_{n, j},
\end{aligned}
$$

where

$$
a_{n k j}(t)=\sum_{l=1}^{n} \varepsilon_{n, k}^{n-1-l} \varepsilon_{n, j}^{l+1} a\left(\varepsilon_{n, l+1} t\right) .
$$

It is easy to check that $P_{n, \nu} K_{b} P_{n, j}=0$ for all $\nu \neq k$ and for $b(t)=a_{n k j}(t)$, which gives $K_{b} X_{j} \subset X_{k}$. The proof is complete.

Corollary 1. Let $j \in\{1, \ldots, n\}$. Then for every $a \in X$ there is a function $b \in X$ that is invariant with respect to the rotation operator $W_{n}$ and

$$
P_{n, j} K_{a} P_{n, j}=K_{b} P_{n, j}=P_{n, j} K_{b} \quad \text { on } X .
$$

Proof. By Lemma 5, we have the equality

$$
P_{n, j} K_{a} P_{n, j}=a_{n j j}(t) P_{n, j},
$$

where

$$
a_{n j j}(t)=\sum_{k=1}^{n} a\left(\varepsilon_{n, k+1} t\right) .
$$

We see from (11) that $a_{n j j}(t)$ is a function invariant with respect to the rotation operator $W_{n}$. Hence $K_{b} P_{n, j}=P_{n, j} K_{b}$ for $b(t)=a_{n j j}(t)$, which was to be proved.

Consider now the equation with the operator $T$ of the form (7):

$$
x(t)+a(t)(T x)(t)=b(t),
$$

where $a, b \in X$ are given.

THEOREM 3. Let $m \in \mathbb{N}^{+}$and $n_{1}, \ldots, n_{m}$ be given distinct positive integers. Suppose that $\mathcal{M}_{n_{j}}(t, t)=1$, and $\mathcal{M}_{n_{j}}(s, t)(j \in\{1, \ldots, m\})$ admit an analytic continuation in both variables into $D^{+}$and are invariant with respect to $W_{N}$, where $N=\prod_{j=1}^{m} n_{j}$. Then the equation (12) has solutions if and only if the equation

$$
y(t)+a^{(m)}(t)\left(\left(S+N_{n_{1}}\right) \ldots\left(S+N_{n_{m}}\right) y\right)(t)=b_{(m)}(t),
$$

where $a^{(m)}(t)$ are constructed by Lemma 4 as follows:

$$
a^{(0)}(t)=a(t), \quad a^{(1)}(t)=a_{n_{1} k_{1} k_{1}}^{(0)}(t), \ldots, a^{(m)}(t)=a_{n_{m} k_{m} k_{m}}^{(m-1)}(t)
$$

and

$$
b_{(m)}(t)=\left(P_{n_{1}, k_{1}} \ldots P_{n_{m}, k_{m}} b\right)(t),
$$

has solutions in the space $X_{(m)}:=P^{\otimes} X$, where $P^{\otimes}=P_{n_{1}, k_{1}} \ldots P_{n_{m}, k_{m}}$. 
Proof. Suppose that (12) has a solution $x_{0}(t)$, i.e.

$$
x_{0}(t)+a(t)\left(T x_{0}\right)(t)=b(t) .
$$

Applying the operator $P^{\otimes}$ to both sides of (15), we obtain $y_{0}(t)=\left(P^{\otimes} x_{0}\right)(t)$ belonging to $X_{(m)}$ and satisfying (13).

Conversely, if $y_{0}(t)$ is a solution in $X_{(m)}$ of $(13)$, then it is easy to check that $x(t)=b(t)-a(t)\left(\left(S+N_{n_{1}}\right) \ldots\left(S+N_{n_{m}}\right) y_{0}\right)(t)$ is a solution of $(12)$, which gives the proof.

Lemma 6. Suppose that $\mathcal{M}_{n_{j}}(s, t)(j \in\{1, \ldots, m\})$ satisfy all assumptions of Theorem 3. Then equation (13) has solutions in $X_{(m)}$ if and only if it has solutions in $X$. Moreover, if $y(t) \in X$ is a solution of (13), then $y_{(m)}(t)=\left(P^{\otimes} y\right)(t)$ is a solution of $(13)$ in $X_{(m)}$.

Proof. By Corollary $1, a^{(m)}(t)$ is invariant with respect to all rotation operators $W_{n_{j}}$ and by (5), we see that every operator $S+N_{n_{j}}$ commutes with any rotation operator $W_{n_{l}}$. Then $P^{\otimes}$ commutes with every $S+N_{n_{j}}$. Hence, if $y \in X$ is a solution of (13), then applying the operator $P^{\otimes}$ to both sides of $(13)$, we see that again $y_{(m)}(t)=\left(P^{\otimes} y\right)(t)$ is also a solution of (13). The proof is complete.

R e m a r $\mathrm{k}$. Lemma 6 shows that it is enough to solve the equation (13) in a given space $X=H^{\mu}(\Gamma)$.

Lemma 7. Let $m$ be an odd positive integer. Suppose that $\mathcal{M}_{n_{j}}(s, t)(j \in$ $\{1, \ldots, m\})$ satisfy all assumptions of Theorem 3 and the function

$$
\mathcal{R}_{m}(s, t)=\frac{a^{(m)}(t)}{1+a^{(m)}(t)} \sum_{k=1}^{m}(-1)^{k+1} \mathcal{N}_{n_{k}}(s, t),
$$

where

$$
\mathcal{N}_{n_{k}}(s, t)=\frac{\mathcal{M}_{n_{k}}(s, t)-1}{s-t}
$$

admits an analytic continuation in both variables in $D^{+}$. Then every solution $y(t) \in X$ of the equation (13) is given by the formula

$$
y(t)=y^{+}(t)+y^{-}(t), \quad y^{-}(t)=\psi^{-}(t), \quad y^{+}(t)=\psi^{+}(t)-\left(R_{m} \psi^{-}\right)(t),
$$

where

$$
\left(R_{m} x\right)(t)=\frac{1}{\pi i} \int_{\Gamma} \mathcal{R}_{m}(s, t) x(s) d s
$$

and $\left(\psi^{+}(t), \psi^{-}(t)\right)$ is a solution of the following Riemann boundary value problem:

$$
\psi^{+}(t)=\frac{a^{(m)}(t)-1}{a^{(m)}(t)+1} \psi^{-}(t)+\frac{b_{(m)}(t)}{1+a^{(m)}(t)} .
$$


Proof. By Lemma 4, the equation (13) is equivalent to the equation

$$
y(t)+a^{(m)}(t)\left(\left(S+\sum_{k=1}^{m}(-1)^{k+1} N_{n_{k}}\right) y\right)(t)=b_{(m)}(t) .
$$

Write $y^{+}(t)=(P y)(t)$ and $y^{-}(t)=(Q y)(t)$. Using the notations (16)-(17), we can rewrite $(19)$ in the form

$$
y^{+}(t)+\frac{1-a^{(m)}(t)}{1+a^{(m)}(t)} y^{-}(t)+\left(R_{m} y\right)(t)=\frac{b_{(m)}(t)}{1+a^{(m)}(t)} .
$$

The assumption on $\mathcal{R}_{m}(s, t)$ implies $\left(R_{m} y^{+}\right)(t)=0$ and $\left(R_{m} y^{-}\right)(t) \in X^{+}$ for all $y \in X$. Hence (20) is a Riemann boundary value problem of the form (18), where $\psi^{+}(t)=y^{+}(t)+\left(R_{m} y^{-}\right)(t)$ and $\psi^{-}(t)=y^{-}(t)$. The proof is complete.

Note that all solutions of the Riemann boundary value problem can be found in closed form (see [1], [2], [4]). Consequently, from Lemma 7, we can formulate the following result.

Corollary 2. All solutions of equation (13) can be given in closed form by means of the Riemann boundary value problem (18).

Lemma 8. Let $m$ be an even positive integer. Suppose that $\mathcal{M}_{n_{j}}(s, t)(j \in$ $\{1, \ldots, m\})$ satisfy all assumptions of Lemma 7 . Then every solution $y(t)$ $\in X$ of the equation (13) is given by the formula

$$
y^{-}(t)=\psi^{-}(t), \quad y^{+}(t)=\psi^{+}(t)-\left(R_{m} \psi^{-}\right)(t),
$$

where

$$
\psi(z)=\frac{1}{\pi i} \int_{\Gamma} \frac{b_{(m)}(s)}{1+a^{(m)}(s)} \frac{d s}{s-z} .
$$

Proof. By Lemma 4, (13) is equivalent to the equation

$$
y(t)+a^{(m)}(t)\left(\left(I+\sum_{k=1}^{m}(-1)^{k} N_{n_{k}}\right) y\right)(t)=b_{(m)}(t) .
$$

Using notations (16)-(17), we can rewrite (23) in the form

$$
y(t)+\left(R_{m} y\right)(t)=\frac{b_{(m)}(t)}{1+a^{(m)}(t)} .
$$

The assumption on $\mathcal{R}_{m}(s, t)$ implies $\left(R_{m} y^{+}\right)(t)=0$ and $\left(R_{m} y^{-}\right)(t) \in X^{+}$. Hence, (24) is of the form

$$
\psi^{+}(t)-\psi^{-}(t)=b_{(m)}(t)\left\{1+a^{(m)}(t)\right\}
$$

which gives the formula (21)-(22) for any solution of (13). 
Now we can formulate the main results of Theorem 3 and of Lemmas 6-7 about solutions of the equation (12) in the following form.

THEOREM 4. Let $m$ be an odd positive integer and let $T$ be of the form (7). Suppose that $\mathcal{M}_{n_{j}}(s, t)(j \in\{1, \ldots, m\})$ satisfy all assumptions of Theorem 3. Moreover, suppose that the function $\mathcal{R}_{m}(s, t)$ of the form (16) admits an analytic continuation in both variables into $D^{+}$. Then equation (12) has solutions if and only if the corresponding Riemann boundary value problem (18) has solutions. If that is the case, every solution of (12) is given by the formula

$$
x(t)=b(t)-a(t)\left(\left(S+N_{n_{1}}\right) \ldots\left(S+N_{n_{m}}\right) y\right)(t),
$$

where $y(t)=\psi^{+}(t)-\left(R_{m} \psi^{-}\right)(t)+\psi^{-}(t)$ and $\psi^{ \pm}(t)$ is a solution of the Riemann boundary value problem (18).

Similarly, Theorem 3 and Lemma 8 together imply the following.

TheOREM 5. Let $m$ be an even positive integer and let $T$ be of the form (7). Suppose that $\mathcal{M}_{n_{j}}(s, t)(j \in\{1, \ldots, m\})$ satisfy all assumptions of Theorem 4. Then equation (12) has solutions of the form (25), where $y(t)=\psi^{+}(t)-\left(R_{m} \psi^{-}\right)(t)+\psi^{-}(t)$ and $\psi(z)$ is of the form $(22)$.

Remark 3. Note that the equation (12) in the case of $m$ even is not a singular integral equation. In that case, (12) is a Fredholm integral equation (see Lemma 4).

\section{References}

[1] F. D. Gakhov, Boundary Value Problems, Oxford, 1966 (3rd Russian complemented and corrected edition, Moscow, 1977).

[2] Nguyen Van Mau, Generalized algebraic elements and linear singular integral equations with transformed argument, Wydawnictwa Politechniki Warszawskiej, Warszawa, 1989.

[3] -, Boundary value problems and controllability of linear systems with right invertible operators, Dissertationes Math. 316 (1992).

[4] D. Przeworska-Rolewicz and S. Rolewicz, Equations in Linear Spaces, Monografie Mat. 47, PWN, Warszawa, 1968.

Faculty of Mathematics

University of Hanoi

90 Nguyen Trai, Dongda

Hanoi, Vietnam 